\title{
Modelling, parameter estimation and assessment of partial shading conditions of photovoltaic modules
}

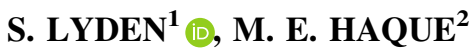

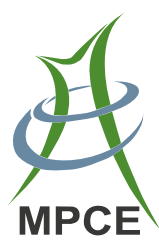

\begin{abstract}
This paper proposes a method for assessing the effect that different features of partial shading conditions (PSC) may have on the operation of a photovoltaic (PV) system. Simulation studies, based on an experimentally validated model of a PV system, are used to assess the influence of PSC. Three classifications of PSC are defined based on the timescale of their influence on the irradiance experienced by the PV module and the relative location of the voltage at which the global maximum power point occurs is assessed. Sample case studies are presented to illustrate the application of the proposed PSC assessment method. The results have implications for the design of future maximum power point tracking methods.
\end{abstract}

Keywords Photovoltaic, Modelling, Partial shading, Maximum power point

CrossCheck date: 19 July 2018

Received: 9 February 2018/Accepted: 19 July 2018/Published online: 20 October 2018

(C) The Author(s) 2018

$\triangle$ S. LYDEN

Sarah.Lyden@utas.edu.au

M. E. HAQUE

enamul.haque@deakin.edu.au

1 School of Engineering, University of Tasmania, Hobart, Australia

2 School of Engineering, Deakin University, Geelong, Australia

\section{Introduction}

Photovoltaic (PV) cells represent a renewable energy source that has highly non-linear characteristics and operation which depends greatly on the environmental conditions. Understanding how the operation of such cells varies under complex and changing environmental conditions is an important step in validating the performance of these systems. PV cells have an optimal operation point which is generally tracked or estimated to ensure efficient operation. Determining this optimum point becomes considerably more complex under non-uniform environmental conditions.

Non-uniform environmental conditions further complicate modelling of the current-voltage (I-V) and powervoltage $(\mathrm{P}-\mathrm{V})$ characteristics of $\mathrm{PV}$ systems. If multiple $\mathrm{PV}$ modules are connected together and one produces less power than the others, this module will limit the power produced by the other modules and may experience hot spot formation or cell damage unless bypass diodes are installed across the modules [1, 2]. Bypass diodes enable the current to flow through an alternative path and skip modules that cannot contribute to the power production. However, the integration of bypass diodes further complicates the $\mathrm{I}-\mathrm{V}$ and $\mathrm{P}-\mathrm{V}$ characteristics as multiple peaks are now observed and the power production may be reduced [3-5]. Non-uniform conditions arising across a module could occur due to shading of part of the module from buildings, trees or other obstacles in the environment, physical damage to the cell, cell ageing over time, or due to the passage of clouds over the module [2, 6, 7]. Especially in the case of cloud movement across the modules, these non-uniform environmental conditions may change very rapidly leading to a considerable reduction in the power extracted from the system. 
Studies completed in the literature to assess the effects of partial shading conditions (PSC) in PV systems take on many different forms. In some studies the position of the $\mathrm{PV}$ modules and obstacles in the environment with respect to the position of the sun at the time of interest are used to map shadows onto the panels [8-14]. Other approaches involve using artificial shading conditions to explore the impact of PSC on the PV characteristics [15-17]. In mapping the shadow onto the modules, some authors $[8,14]$ consider the effects of direct and diffuse irradiance. Defining obstacles by linear functions and a transmission factor for non-opaque objects is suggested by [10]. The 3D mapping of shadows can be achieved using the sun's position and simplified ray tracking with the irradiance on the cell being the averaged shaded and non-shaded irradiance [12]. A PV systems installers guide [11] recommends that close objects provide more direct shading, and therefore a more significant reduction in irradiance, than objects that are far away. The light generated current can be expressed as a function of the shaded area, photo-current density and shadow transmittance to develop PSC [15]. Shading is represented by a shading strength and shading percentage (representing what portion of the system is shaded) by [16], and as a simple percentage by [17]. The shading impacts can also be assessed by considering how the configuration of modules in a system can reduce the effect of shading from obstacles [18] or due to cloud transients [19]. A recent study into the effect of PSC on PV modules has defined five statements which define typical behaviours of PSC [20]. These statements lead to two hypotheses for predicting the number of local maximum power points (MPPs). Other recent work has combined a sky map with a sensitivity map for PV modules that have reflectors to estimate incident irradiance composed of reflected light, direct light and scattered light [21]. These results show good prediction of incident irradiance, however the method has greater complexity than the method adopted in this paper.

The shading analysis presented in this paper is unique as the primary intention of the study is to explore how the relative location of the global maximum power point (GMPP) voltage varies when the shading conditions change. To achieve this objective while minimising computational load, some essential assumptions have been made including using the global direct irradiance rather than separating direct and diffuse irradiance in this preliminary study. Three types of PSC are defined. These are constant, static and transient PSC. Constant PSC represents a reduction in irradiance that will always be present, such as due to cell ageing or damage. Static PSC represents shading which changes slowly with time, such as the shade from an object in the environment which may also have a shading strength associated with it. Transient PSC is much quicker and represents the irradiance at a particular point based on the time of day and cloud cover. Essentially transient PSC can be considered as the instantaneous irradiance reaching the Earth's surface which may vary very rapidly based on cloud cover. As a residential scale system is considered in this paper, the geographical area of the system is small such that transient PSC (or the incident irradiance from the sun) is assumed constant across all modules in the system at any point in time. The irradiance on each module is determined by considering the constant shading factor, static shading factor and transient irradiance.

The classification of shading types considered in this paper is more comprehensive than that presented by [22] as it also considers the changing irradiance due to cloud cover and time of day as a type of shading phenomenon. The approach of using $1 \mathrm{~min}$ solar data and matrices to provide a shading factor for each module to model the different shading on modules has been considered by [23] however, in their paper the main shading effects considered relate more so to transient PSC defined above. The purpose of the study presented in this paper is to enable the effects of constant, static and transient PSC on the location of the GMPP voltage to be isolated and quantified. This has direct implications for the design of future global maximum power point tracking (GMPPT) methods.

The main contributions of this paper include:

1) Defining three classifications of PSC and utilising these to isolate the individual impacts of shading on a PV system.

2) Development of a method and case studies for assessing the effects of constant, static and transient PSC on the location of the GMPP voltage.

3) Identification of the implications this study has for GMPPT.

Section 2 presents a method for assessing the impact of the three PSC types defined above on the relative location of the GMPP based on an experimentally validated PV module simulation model. In Section 3, three sample case studies are presented to show how the PSC assessment method can be used with simulated obstacles and constant cell ageing factor related partial shading on the PV modules. Finally, Section 4 discusses the key observations from the case studies and possible implications for MPPT and Section 5 presents the paper conclusions.

\section{Modelling and assessment of partial shading conditions}

In this paper, the effects of partial shading are considered based on a simulation model made up of eight series-connected BP380 PV module using the single diode model 
Table 1 Parameters of BP380 PV module

\begin{tabular}{lr}
\hline Parameter & Value \\
\hline$V_{o c}$ & $22.10 \mathrm{~V}$ \\
$I_{s c}$ & $4.80 \mathrm{~A}$ \\
$V_{m p p}$ & $17.60 \mathrm{~V}$ \\
$I_{m p p}$ & $4.55 \mathrm{~A}$ \\
$P_{m p p}$ & $80.10 \mathrm{~W}$ \\
$N_{s}$ & 36 \\
\hline
\end{tabular}

(SDM). The parameters of the BP380 PV module are given in Table 1 below [24]. Based on its acceptable accuracy $[25,26]$ the SDM is used in this paper to enable complex PV systems to be simulated with less computational complexity. The three levels of partial shading are defined as constant, static and transient partial shading. These definitions of PSC separate effects that will always be constant or changing so slowly that they will have limited impact on a day of operation of the system, from those that change very rapidly such as due to the time of day and cloud cover. Constant PSC represents a reduction in irradiance that will always be present, such as due to cell ageing. Static PSC represents shading which changes slowly with time, such as the shade from an object in the environment. Transient PSC is much quicker and represents the irradiance at a particular point based on the time of day and cloud cover. The purpose of dividing partial shading into three distinct effects is to explore which of these is the most significant in influencing the relative location of the GMPP voltage and use this to inform the future development of GMPPT strategies.

In this paper residential scale PV systems are of interest and are of sufficiently small size that the transient shading factor at any point in time can be assumed constant across all the modules in the system. Transient PSC is the shading due to changing irradiance level and is represented in this study through $1 \mathrm{~min}$ irradiance data from the Australian Bureau of Meteorology (BOM) [27]. This idea of transient PSC being modelled by 1 min data is supported by [28], who determined that shading periods caused by clouds have an average duration of $60 \mathrm{~s}$, but may range from $4 \mathrm{~s}$ up to $1.5 \mathrm{~h}$. Constant PSC can be modelled by applying a constant shading factor to the module. Static partial shading is modelled by placing a simulated obstacle in the environment and modelling the shadow from the object based on the position of the sun in the sky.

The module is considered shaded if the shadow from the object overlaps with the defined module location. The number of cells in the module which experience shading due to an obstacle define a shading factor for that module. The irradiance on the module is found by multiplying this shading factor by the constant shading factor and the transient shading from the $1 \mathrm{~min}$ irradiance data. If there is no shading of any of the cells in the module the shading factor is 1 , and if all cells are shaded the shading factor is 0 . For all other shading conditions the shading factor lies between 0 and 1 and is calculated using (1), where $N_{s}$ is the number of series-connected cells within the module, and shaded-cells represent the number of shaded cells.

$s f=1-\left(\right.$ shaded-cells $\left./ N_{s}\right)$

This paper adopts a simple approach for estimating which cells within the module are shaded by the obstacle. A simple approach is deemed appropriate as the key objective of the study is to develop a preliminary set of observations related to how different shading phenomena affect the relative location of the GMPP with respect to its voltage. The number of cells that are shaded by the object are found by considering the object orientation in the environment and calculating the shadow tip position for the time and day of the year. Objects define their own coordinate basis, such that each object is located at coordinates $(0,0)$ on the north-east axis, and the distance to the closest corner of the PV module is defined. In this coordinate system it is possible to determine when the cells of each module lie within half of the object width of the shadow centre line (defined by joining the object origin to the shadow tip) indicating that they are most likely shaded. For simplicity, the width of the shadow is assumed equal to the object width. Complex shadow shapes are not considered in this analysis and the global direct irradiance is applied without separating the impacts of direct and diffuse irradiance. These simplifications are deemed appropriate as the primary goal of the study is to investigate the movement of the relative voltage of the GMPP as authentic shading conditions change.

The series of equations given below provides a process for evaluating the shadow tip location $[9,29,30]$, where $\phi$ denotes latitude; $\delta$ denotes declination angle; $\alpha$ denotes elevation angle; $\omega$ denotes longitude angle; $L T$ denotes local time; $N$ denotes day of the year; $\Delta T_{G M T}$ denotes difference of local time from GMT in hours $(10 \mathrm{~h}$ in Tasmania).

The local standard time meridian $L S M$ is:

$L S M=15^{\circ} \Delta T_{G M T}$

The equation of time EoT can be given by:

$E o T=9.87 \sin 2 B-7.53 \cos B-1.5 \sin B$

where $B$ is given by:

$B=\frac{360}{365}(N-81)$

The time correction $T C$ is:

$T C=4(\omega-L S M)+E o T$

which provides a local solar time $L S T$ : 
$L S T=L T+\frac{T C}{60}$

The hour angle $H R A$ can be found from:

$H R A=15^{\circ}(L S T-12)$

The elevation angle can be evaluated from:

$\alpha=\arcsin [\sin \delta \sin \phi+\cos \delta \cos \phi+\cos H R A]$

The azimuth can be calculated as the following. Note that Azimuth gives the direction of the sun. And Azi is a temporary value as the actual azimuth needs to be calculated slightly different depending on $H R A$.

$A z i=\operatorname{arcos} \frac{\sin \delta \sin \phi-\cos \delta \sin \phi \cos H R A}{\cos \alpha}$

Azimuth $= \begin{cases}A z i & \text { if } H R A<0 \\ 360^{\circ}-A z i & \text { if } H R A>0\end{cases}$

The shadow length can be found from

$W=\frac{H}{\tan \alpha}$

where $H$ is the height of the object in the environment.

Finally the shadow tip location in terms of $x$ and $y$ coordinates can be calculated from the azimuth and elevation angle for each minute at the location of interest using (12).

$\left\{\begin{array}{l}x=W \frac{\sin \left(\text { Azimuth }-180^{\circ}\right)}{\tan \alpha} \\ y=W \frac{\cos \left(\text { Azimuth }-180^{\circ}\right)}{\tan \alpha}\end{array}\right.$

Once the shadow path of an obstacle is known it can be used to determine the static shading factor on each module in the system using (1). This shading factor is then multiplied by the constant and transient shading factors to establish an estimate of the equivalent irradiance on each module. This can then be used with the eight series-connected PV modules model to develop a $\mathrm{P}-\mathrm{V}$ curve trace for the system showing the impact of PSC at that particular instant in time. This process is repeated across a sample of shading and irradiance conditions to monitor how these changes impact on the relative location of the GMPP in terms of voltage. These sample conditions are based on modelling objects in the system at a particular location on a particular date and time, and utilising BOM 1 min known irradiance data that corresponds with that same time, date and location.

The procedure adopted for modelling and assessment of PSC under a variety of different conditions is given in Fig. 1. $P_{g m p p}$ is the power at the global maximum power point and $V_{g m p p}$ is the voltage at the global maximum power point. This procedure is generic and relies on the equations defined in this section. In the case studies presented in Sect. 3, 1 min solar irradiance data has been used, so each characteristic is drawn on a 1 min basis.

\section{Partial shading case studies}

Four case studies on the eight series-connected modules simulation model from Sect. 2 are explored in this section. These case studies represent a subset of the extensive additional case studies completed and highlight the key findings from the other cases. Other case studies have been omitted due to space requirements. In the first case study there is only one obstacle located in the environment and in the second case study there are two obstacles in the environment. Case 3 involves a constant PSC being applied to the system to represent cell ageing and damage. Case 4 incorporates a shading strength into the calculation of shading factor for two obstacles in the environment. The simulation model enables the location of the voltage of the GMPP to be monitored and key observations on the transitions of the GMPP location under constant, static and transient PSC can be described for each case study.

\subsection{Case 1}

In case study 1 , one obstacle is placed in the environment, as shown in Fig. 2. The obstacle is $20 \mathrm{~m}$ in height and $0.25 \mathrm{~m}$ in width, and may be representative of an

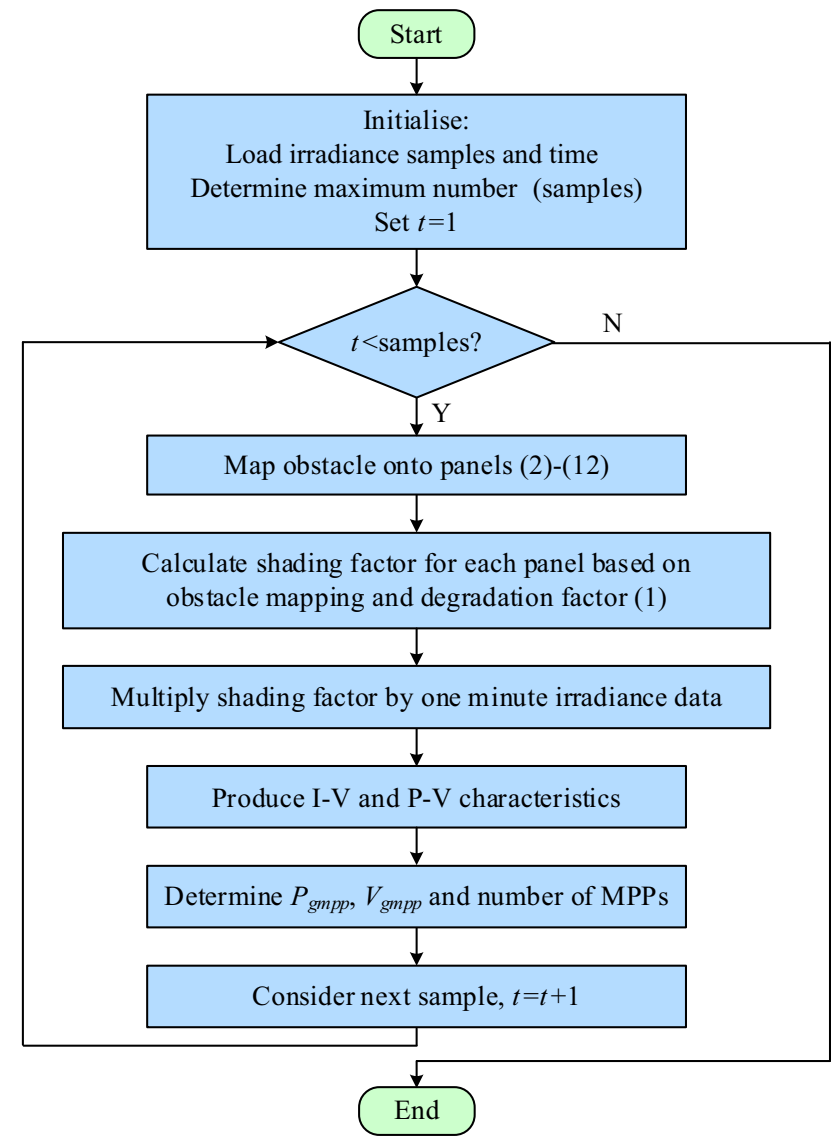

Fig. 1 Partial shading modelling and assessment process 
obstacle such as a power pole or thin tree in the nearby environment. For the irradiance data of interest in 2010, shading from the object occurs over the PV system from 28 February to 11 October producing 222 days and $15655 \mathrm{~min}$ where the PV modules experiences shading. The P-V characteristics across the day for 20 March 2010, represented as a 3D surface, are shown in Fig. 3. This picture represents a small sample of the information collected in this case study. Information regarding the GMPP voltage and power across all minutes of shading are recorded to enable key characteristics of the movement of the GMPP voltage to be assessed. The GMPP voltage and power can be extracted from this three dimensional characteristic and used to determine the change in relative GMPP location from each minute to the next. The transitions in GMPP power and voltage across the 58 min sample are shown in Figs. 4 and 5, respectively. A transition occurs when from one irradiance and shading condition to the next the relative location of the GMPP in terms of voltage varies substantially. It is suggested that the MPPs on the P-V characteristic are separated by approximately $0.8 V_{o c}$, where $V_{o c}$ is the open circuit of the module [2]. Using this as a guide, the $\mathrm{P}-\mathrm{V}$ characteristic exhibits regions that could correspond to MPP1 to MPP8 for the eight module system, based on the fact that each module in the system could experience a different level of irradiance determined by shading conditions. In this analysis, the purpose is to monitor the number of minutes across the shaded time when the GMPP is in each MPP location and to assess the transitions that occur between the MPP locations when a change in the environmental conditions occurs. For this case study, Tables 2 and 3 demonstrate the transitions and MPP locations, respectively. For each quantity the average, minimum, maximum, standard deviation and count is indicated.

From these tables, it can be seen that it is most likely that the GMPP voltage will remain around the same MPP region $(77.85 \%)$ or transition to an adjacent MPP region $(22.06 \%)$ between each minute of the study. In a very small number of cases $(0.1 \%)$ a transition to a MPP region further away was observed. It can also be seen that during the

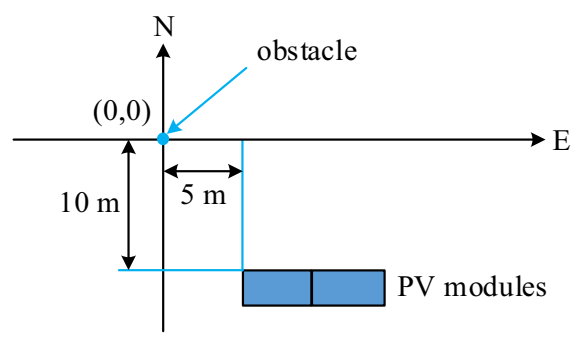

Fig. $2 \mathrm{XY}$ representation of obstacle and PV modules in the environment for Case 1

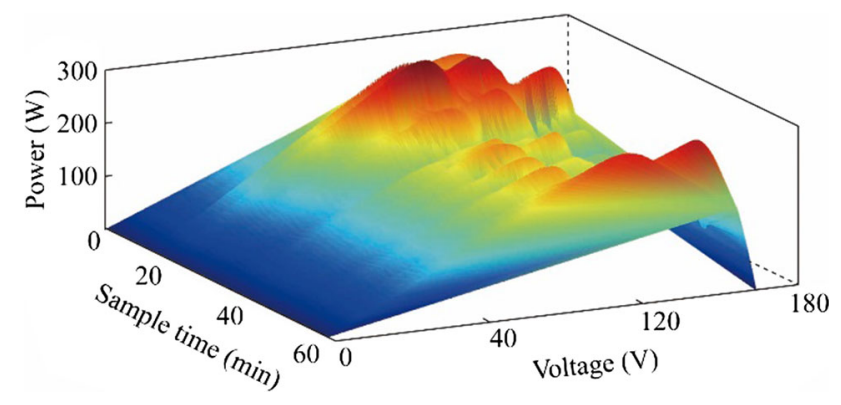

Fig. 3 Sample 3D representation of $\mathrm{P}-\mathrm{V}$ characteristics for sample day taken from 20 March 2010 where there are 58 min of shading

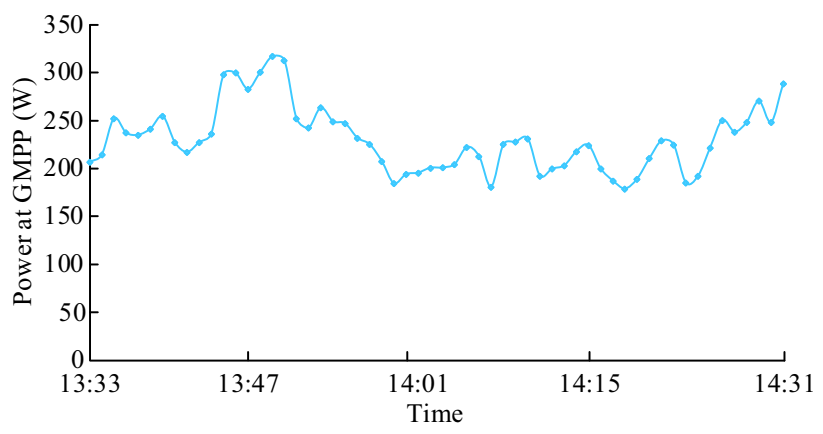

Fig. 4 Trends in GMPP power for 20 March 2010 during the 58 min of shading

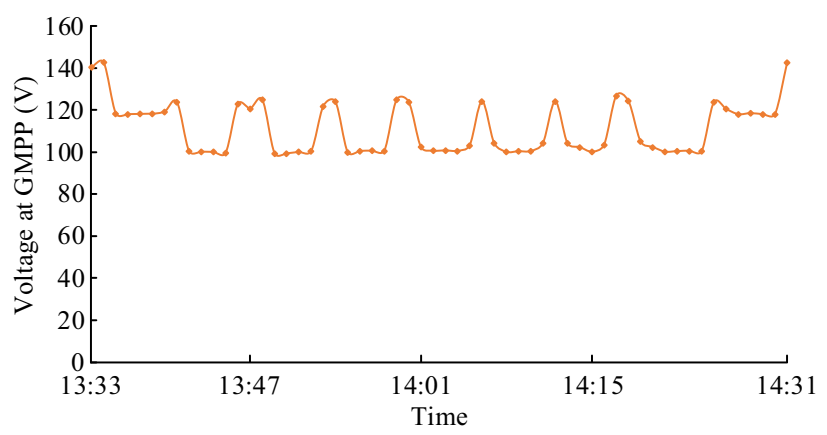

Fig. 5 Trends in the voltage at the GMPP for 20 March 2010 during the 58 min of shading

shading time, the GMPP was most likely in the region of MPP6 (51.63\%).

\subsection{Case 2}

In case study 2, two obstacles are placed in the environment as shown in Fig. 6. The same obstacle from Case 1 is used with an additional $1 \mathrm{~m}$ tall, $0.25 \mathrm{~m}$ wide obstacle placed much closer to the PV system, which could represent a chimney on the roof where the PV system is installed. Using the study irradiance data from 2010, shading occurs from 4 February to 31 October, producing 263 days and $68513 \mathrm{~min}$ of shading data. 
Table 2 Transitions for Case 1

\begin{tabular}{lllllr}
\hline Transition & Average & Minimum & Maximum & Standard deviation & Count \\
\hline One MPP transition & 15.55 & 0 & 18 & 2.12 & 3453 \\
Transition of two MPPs & 0.07 & 0 & 5 & 0.41 & 15 \\
\hline
\end{tabular}

Table 3 MPP locations for Case 1

\begin{tabular}{lllllr}
\hline Location & Average & Minimum & Maximum & Standard deviation \\
\hline MPP6 & 36.40 & 0 & 60 & 10.50 & 8083 \\
MPP7 & 29.64 & 0 & 41 & 6.62 & 6580 \\
MPP8 & 4.47 & 2 & 16 & 1.97 & 992 \\
\hline
\end{tabular}

The $\mathrm{P}-\mathrm{V}$ characteristics across the day for 20 March 2010 as a 3D representation are shown in Fig. 7. The transitions and the MPP locations for Case 2 are given in Tables 4 and 5, respectively. It can be seen that the GMPP voltage will most likely remain around the same MPP region $(92.08 \%)$ or move to an adjacent MPP region (4.84\%). Other transitions become less likely as the change in voltage required to move between the regions increases. During the shading time, the MPP is more likely to be at MPP8 (39.68\%), followed by MPP7 (27.83\%), MPP6 (17.12\%), and MPP5 (13.93\%). Compared to Case 1, this suggests that when more complex shading occurs it is likely that the GMPP location will move between more of the MPP regions with respect to voltage.

\subsection{Case 3}

Case 3 involves applying a constant reduction in the irradiance of some modules to represent the effects of cell ageing, damage and mismatch. A subset of the shading data used in Case 1 and Case 2 is used in this case as the trends observed remain fairly consistent across the year. A constant shading factor between 0.90 and 1.00 is applied to each module in the system. Nine days, one from each month between February and October, are considered where shading is studied between 12:00 and 15:59. This produces a data set with 9 days and $2160 \mathrm{~min}$ to enable the effects of constant PSC to be assessed. A 3D representation of the P-V characteristics for 26 March 2010 under constant PSC is shown in Fig. 8. Under constant PSC, from the data collected it can be seen that the GMPP remains at MPP8 and undergoes no transitions to another MPP region. This is because the small amount of degradation for each module, only produces a small variation in the $\mathrm{P}-\mathrm{V}$

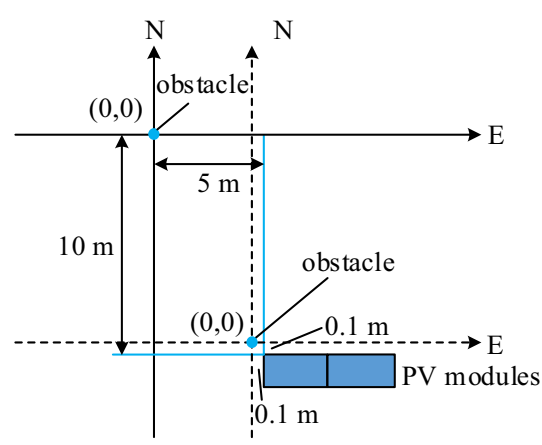

Fig. $6 \mathrm{XY}$ representation of the obstacle and PV modules in the environment for two obstacle

characteristics and not enough to lead to the GMPP moving significantly.

\subsection{Case 4}

In the previous case studies the shading from objects has been assumed to cause the cells in the shadow path to be completely shaded. In reality, objects in the environment, may only reduce the irradiance by some factor rather than reducing it absolutely based on the properties of the object. For example, through the branches of a tree in winter, the irradiance will not be solidly reduced by the basic shape approximation used in this study, but may cause a factor of reduction in the incident irradiance on affected cells. To assess if the relative strength of shading - defined by a factor between 0 and 1, affects the relative location of the GMPP voltage, various shading strengths are considered on a sample day of shading from Case 2. The day of study is 23 May 2010, and the shading strength is applied to the existing 264 min of shading caused by the two obstacles from Case 2. The new shading factor is determined by: 
$s f=1-\frac{\text { shaded-cells } \times \text { shaded-strength }}{N_{s}}$

Shading strengths of $0.2,0.5$, and 0.7 are applied to the shading scenario of Case 2 on 23 May 2010 to assess if this factor has any influence on the relative location of the GMPP voltage.

The results show that shading strength has a direct effect on the shading factor for each module in the system. Figure 9 shows a selection of shading factors per module for each of the shading strengths considered. The darker red colour indicates a smaller value of shading factor (i.e. lower irradiance on the module). Here the colouring indicates the shading factor, where green is a shading factor of 1 , and the colours transition through white to dark red as the shading factor becomes smaller (i.e. as the module experiences greater shading). Clearly by comparing these scenarios side by side, it can be seen that the change in shading strength has not influenced which modules are shaded at any given point in time, but does influence the value of the shading factor.

By changing the shading factor some slight changes in the P-V characteristics can be observed. Increasing the shading strength leads to a lower GMPP power across all samples, as is shown in Fig. 10. The relative change in shading factor between the samples due to the shading

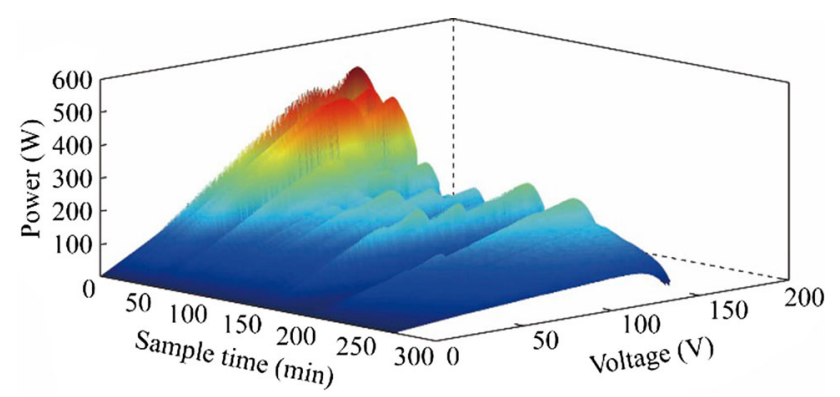

Fig. 7 Sample 3D representation of P-V characteristics for 20 March 2010 during 265 min of shading for Case 2 strength may result in a change in the GMPP voltage as shown in Fig. 11. Based on Fig. 11, it can be seen that as the shading strength increases to 0.7 the number of one MPP region and two MPP region transitions increases to 8 and 13 , respectively. This is compared with the shading strength 0.5 which has 6 one MPP region transitions and 1 two MPP region transition, and shading strength of 0.2 which has 2 one MPP region transitions during the sample case.

These results show that the shading factor has no influence on the time of shading as the path of the obstacle across the modules is fixed. The shading factor however works with static shading to change the movement of the GMPP and reduces the overall power available as the shading factor increases. As the shading strength increases, the shading becomes more severe leading to a greater number of transitions between adjacent MPP locations.

\section{Key observations}

Key observations that can be made from the case studies presented in this paper include:

1) Power available at the GMPP is most affected by the transient PSC (the irradiance).

2) With constant PSC small local peaks occur in the PV characteristics however the GMPP voltage is not influenced significantly.

3) The most significant factor in moving the relative location of the GMPP with respect to voltage is static partial shading, resulting from the movement of shadows from obstacles in the environment.

4) Shading strength influences the shading factor caused by static shading but only when the static obstacle shadow maps onto the modules.

5) As shading strength increases, the likelihood of the GMPP voltage moving to an adjacent region increases based on the corresponding more significant change in shading factor in the modules of the system.

Table 4 Transitions for Case 2

\begin{tabular}{|c|c|c|c|c|c|}
\hline Transition & Average & Minimum & Maximum & Standard deviation & Count \\
\hline One MPP transition & 12.61 & 2 & 26 & 4.67 & 3316 \\
\hline Transition of two MPPs & 4.30 & 0 & 11 & 2.63 & 1130 \\
\hline Transition of three MPPs & 3.26 & 0 & 8 & 2.43 & 858 \\
\hline Transition of four MPPs & 0.60 & 0 & 4 & 0.92 & 124 \\
\hline Transition of five MPPs & 0.01 & 0 & 1 & 0.10 & 1 \\
\hline
\end{tabular}


Table 5 MPP locations for Case 2

\begin{tabular}{lrlcrr}
\hline Location & Average & Minimum & Maximum & Standard deviation & Count \\
\hline MPP2 & 0.01 & 0 & 1 & 0.10 & 9.06 \\
MPP4 & 4.62 & 0 & 62 & 966 \\
MPP5 & 36.30 & 0 & 80 & 22.49 & 9546 \\
MPP6 & 44.60 & 0 & 146 & 20.41 & 11730 \\
MPP7 & 72.50 & 1 & 227 & 23.14 & 19068 \\
MPP8 & 103.37 & 7 & 61.00 & 27187 \\
\hline
\end{tabular}

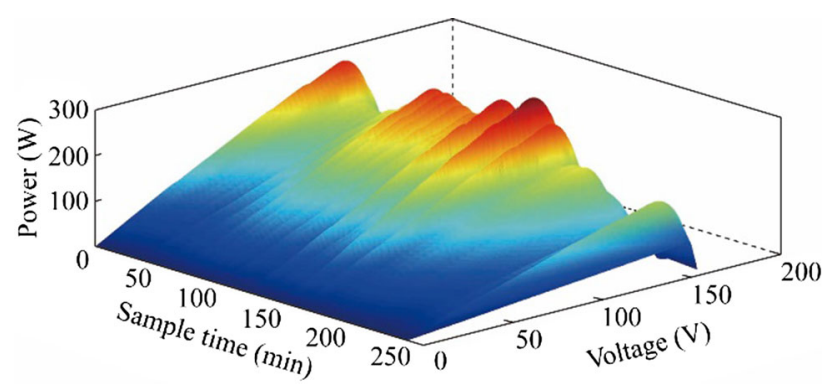

Fig. 8 Sample 3D representation of P-V characteristics for 26 March 2010 during 239 min of shading for Case 3

6) The GMPP is more likely to remain around the same MPP region with respect to voltage or move to an adjacent MPP region, rather than undergoing a transition across several MPP regions.

The method proposed in this paper has also been applied with $1 \mathrm{~s}$ irradiance data available from NREL [31] demonstrating similar results. These results are not shown in this paper due to space restrictions, but are supported by the fact that shading periods caused by clouds have an average duration of $60 \mathrm{~s} \mathrm{[28]} \mathrm{and} \mathrm{the} \mathrm{relative} \mathrm{location} \mathrm{of}$ the GMPP in terms of voltage is more sensitive to the relatively static PSC arising from obstacle placement in the environment which will not substantially change from $1 \mathrm{~s}$ to the next.

These key observations have a direct implication for the future development of GMPPT methods. In particular, these results suggest that when trying to locate a new maxima it may be possible to search within a much smaller neighbourhood of the previous operating point. This is indicated by the representative results included in this paper and supported by the other case studies and sets of data omitted due to space, which show that in the presence of shading from an obstacle, the GMPP relative location is most likely to remain at the same point or transition to an adjacent MPP location. This significantly reduces the search range when a change in conditions is detected and provides a mechanism for monitoring adjacent peaks [2] as a method of detecting a change in the static shading situation [32]. The results also show that when small scale

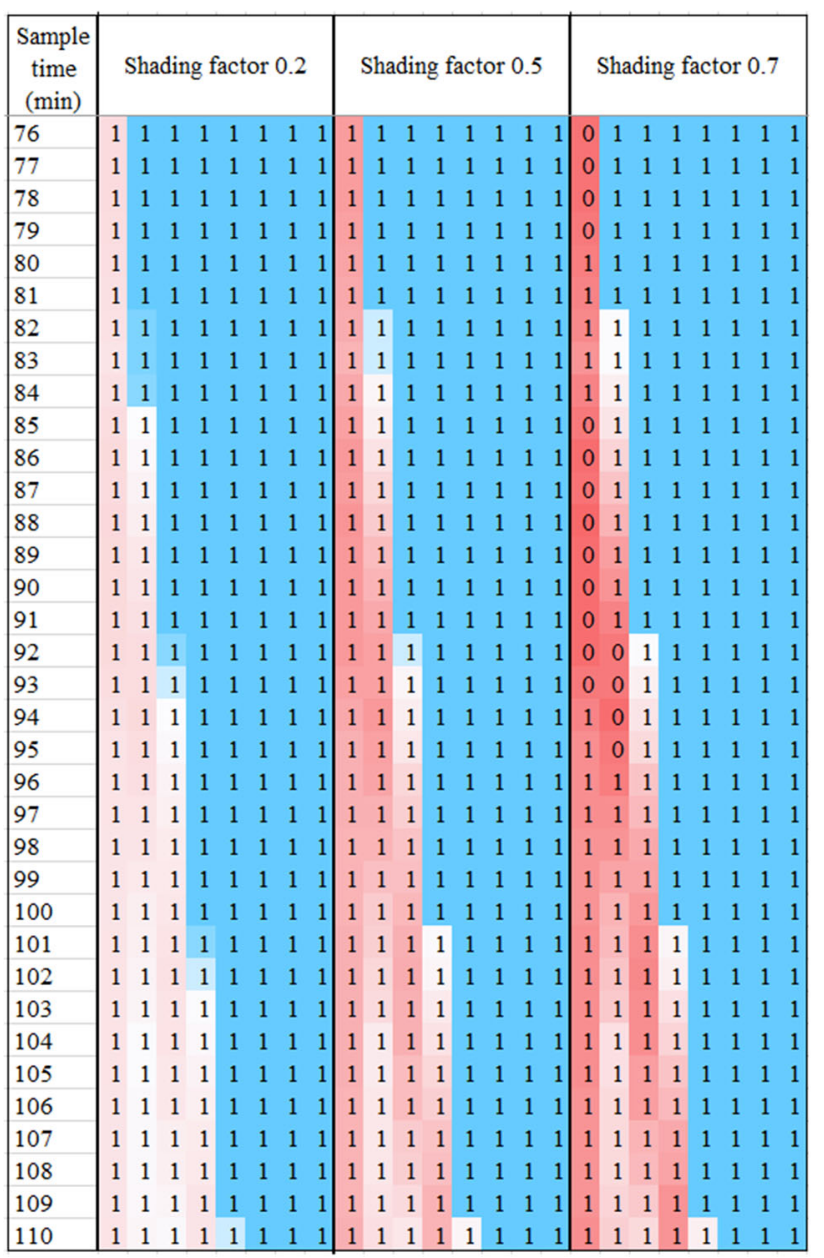

Fig. 9 Sample of influence of shading strength on the shading factor for the 8 module PV system with two obstacles in the environment

residential PV systems are considered, static partial shading caused by objects in the environment will have the most significant impact on the relative location of the GMPP while the transient PSC will have the greatest effect on the power available. By isolating the effects of the different types of PSC, the effects that each of these will have on a particular PV system can be estimated before the system is installed. By modelling the movement of the shadow of obstacles across the modules, it is possible to 


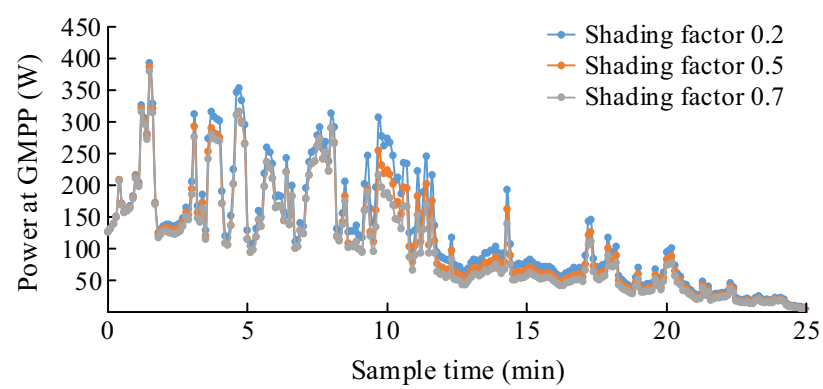

Fig. 10 Trends in GMPP power for 23 May 2010 with 2 obstacles in environment and different shading strengths

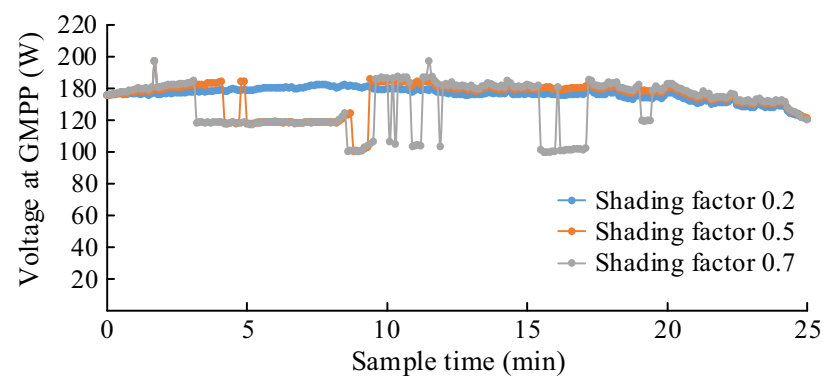

Fig. 11 Trends in GMPP voltage for 23 May 2010 with 2 obstacles in environment and different shading strengths

select an optimal configuration of the modules to minimise PSC losses.

\section{Conclusion}

In this paper a PSC assessment strategy has been proposed and explored through several case studies. These case studies show that the shading from objects in the environment has the greatest effect on the relative location of the GMPP voltage when compared with constant cell degradation and rapidly varying irradiance. The results have also shown that typically when the GMPP voltage moves to another region due to a change in environmental conditions, this is most commonly to an adjacent position. This has implications for the design of GMPPT strategies as it provides guidance on a suitable searching range of the previous operating point when attempting to locate a new GMPP.

Open Access This article is distributed under the terms of the Creative Commons Attribution 4.0 International License (http:// creativecommons.org/licenses/by/4.0/), which permits unrestricted use, distribution, and reproduction in any medium, provided you give appropriate credit to the original author(s) and the source, provide a link to the Creative Commons license, and indicate if changes were made.

\section{References}

[1] Keyhani A (2011) Design of smart power grid renewable energy systems. Wiley, New York

[2] Patel H, Agarwal V (2008) MATLAB-based modeling to study the effects of partial shading on PV array characteristics. IEEE Trans Energy Convers 23(1):302-310

[3] Moballegh S, Jiang J (2011) Partial shading modeling of photovoltaic system with experimental validations. In: Proceedings of 2011 IEEE power and energy society general meeting, Detroit, USA, 24-29 July 2011, 9 pp

[4] Alajmi BN, Ahmed KH, Finney SJ et al (2013) A maximum power point tracking technique for partially shaded photovoltaic systems in microgrids. IEEE Trans Ind Electron 60(4):1596-1606

[5] Hassan R, Radman G, Gao D (2011) User-friendly LabView tool to study effects of partial shading on PV characteristics. In: Proceedings of 2011 proceedings of IEEE Southeastcon, Nashville, USA, 17-20 March 2011, 4 pp

[6] Ji YH, Kim JG, Park SH et al (2009) C-language based PV array simulation technique considering effects of partial shading. In: Proceedings of 2009 IEEE international conference on industrial technology, Gippsland, Australia, 10-13 February 2009, 6 pp

[7] Attivissimo F, Nisio AD, Savino M et al (2012) Uncertainty analysis in photovoltaic cell parameter estimation. IEEE Trans Instrum Meas 61(5):1334-1342

[8] Quaschning V, Hanitsch R (1995) Shade calculations in photovoltaic systems. In: Proceedings of ISES solar world conference, Harare, Zimbabwe, 11-15 September 1995, 5 pp

[9] Jazayeri K, Uysal S, Jazayeri M (2013) MATLAB/simulink based simulation of solar incidence angle and the sun's position in the sky with respect to observation points on the Earth. In: Proceedings of 2013 international conference on renewable energy research and applications (ICRERA), Madrid, Spain, 20-23 October 2013, 5 pp

[10] Drif M, Pérez PJ, Aguilera J et al (2008) A new estimation method of irradiance on a partially shaded PV generator in gridconnected photovoltaic systems. Renew Energy 33(9): 2048-2056

[11] für Sonnenenergie DG (2013) Planning and installing photovoltaic systems: a guide for installers, architects and engineers. Routledge, London

[12] MacAlpine SM, Erickson RW, Brandemuehl MJ (2013) Characterization of power optimizer potential to increase energy capture in photovoltaic systems operating under nonuniform conditions. IEEE Trans Power Electron 28(6):2936-2945

[13] Sinapis K, Tzikas C, Litjens G et al (2016) A comprehensive study on partial shading response of c-Si modules and yield modeling of string inverter and module level power electronics. Solar Energy 135:731-741

[14] Goss B, Cole I, Betts T et al (2014) Irradiance modelling for individual cells of shaded solar photovoltaic arrays. Solar Energy 110:410-419

[15] Guo S, Walsh TM, Aberle AG et al (2012) Analysing partial shading of PV modules by circuit modelling. In: Proceedings of 2012 38th IEEE photovoltaic specialists conference, Austin, USA, 3-8 June 2012, 4 pp

[16] Mäki A, Valkealahti S, Leppäaho J (2012) Operation of seriesconnected silicon-based photovoltaic modules under partial shading conditions. Prog Photovolt Res Appl 20(3):298-309

[17] Wang YJ, Hsu PC (2009) Analysis of partially shaded PV modules using piecewise linear parallel branches model. World Acad Sci Eng Technol 60:783-789 
[18] Belhachat F, Larbes C (2015) Modeling, analysis and comparison of solar photovoltaic array configurations under partial shading conditions. Solar Energy 120:399-418

[19] Lappalainen K, Valkealahti S (2017) Effects of irradiance transition characteristics on the mismatch losses of different electrical PV array configurations. IET Renew Power Gener 11(2):248-254

[20] Ahmad R, Murtaza AF, Sher HA et al (2017) An analytical approach to study partial shading effects on PV array supported by literature. Renew Sustain Energy Rev 74:721-732

[21] Santbergen R, Muthukumar VA, Valckenborg RME et al (2017) Calculation of irradiance distribution on PV modules by combining sky and sensitivity maps. Solar Energy 150:49-54

[22] Hariharan R, Chakkarapani M, Ilango GS et al (2016) A method to detect photovoltaic array faults and partial shading in PV systems. IEEE J Photovolt 6(5):1278-1285

[23] Severini M, Principi E, Fagiani M et al (2017) Energy management with support of PV partial shading modelling in micro grid environments. Energies 10(4):453

[24] BP $38080 \mathrm{~W}$ photovoltaic module datasheet. http://www. solarcharge.com.au/docus/off-grid-solar-panel-BP-80-Watt.pdf. Accessed 31 Mar 2012

[25] Villalva MG, Gazoli JR, Filho ER (2009) Comprehensive approach to modelling and simulation of photovoltaic arrays. IEEE Trans Power Electron 24(5):1198-1208

[26] Chatterjee A, Keyhani A (2011) Thevenin's equivalent of photovoltaic source models for MPPT and power grid studies. In: Proceedings of IEEE power and energy society general meeting, Detroit, USA, 24-29 July 2011, 7 pp

[27] Bureau of Meteoroloy (2012) One minute solar data. http:// www.bom.gov.au/climate/data/oneminsolar/about-IDCJAC0022. shtml. Accessed 13 Nov 2013

[28] Lappalainen K, Valkealahti S (2016) Analysis of shading periods caused by moving clouds. Solar Energy 135:188-196
[29] Honsberg C, Bowden S (2014) The sun's position/PVEducation. http://www.pveducation.org/pvcdrom/properties-ofsunlight/sunsposition. Accessed 24 January 2017

[30] Iqbal M (2012) An introduction to solar radiation. Elsevier, New York

[31] National Renewable Energy Laboratory (2014) Oahu solar measurement grid: 1-second global horizontal irradiance. http:// www.nrel.gov/midc/oahu_archive/. Accessed 30 June 2014

[32] Lyden S, Haque ME (2016) A simulated annealing global maximum power point tracking approach for PV modules under partial shading conditions. IEEE Trans Power Electron 31(6):4171-4181

S. LYDEN received the Graduate degree with a B.Sc.-B.E. (Hons.) and the Ph.D. degree in engineering from the University of Tasmania, Hobart, Australia, in 2011 and 2015, respectively. She is currently a Lecturer at the School of Engineering, University of Tasmania. Her research interests include control and grid integration of renewable energy sources, microgrid systems, and engineering education.

M. E. HAQUE received the Graduate degree in Electrical and Electronic Engineering from the Rajshahi University of Engineering Technology (formerly, the Bangladesh Institute of Technology), Rajshahi, Bangladesh, in 1995, the M.E. degree in electrical engineering from University Technology Malaysia, Kuala Lumpur, Malaysia, in 1998, and the Ph.D. degree in electrical engineering from the University of New South Wales, Sydney, Australia, in 2002. He is currently a Senior Lecturer in renewable energy and power systems at the School of Engineering, Deakin University, Geelong, Australia. His research interests include smart energy systems, control and grid integration of renewable energy sources and energy storage systems, microgrid system with hybrid wind/solar/fuel cell systems, and power electronics applications in smart grid and renewable energy systems. 